\title{
Little evidence exists about optimal caries-prevention strategies during orthodontic treatment
}

\author{
What is the most effective method of preventing white spot lesions during \\ orthodontic treatment?
}

\begin{abstract}
Derks A, Katsaros C, Frencken JE, van't Hof MA, KuijpersJagtman AM. Caries-inhibiting effect of preventive measures during orthodontic treatment with fixed appliances. A systematic review. Caries Res 2004; 38:413-420
\end{abstract}

Data sources Medline, PubMed and hand searches were used to source studies.

Study selection Publications were screened independently by two observers. Only randomised controlled trials (RCT) of orthodontic fixed appliance treatment with bonded brackets that addressed prevention of white spot lesions which were published in English were included. Studies also had to provide enough data to calculate the preventive fraction (PF).

Data extraction and synthesis The studies were divided into four groups based on comparable preventive measures (fluoride, chlorhexidine, sealants and bonding materials) and then the PF was calculated. Results The overall PF of the fluoride-releasing bonding materials was $20 \%$ (standard error, 0.09). This effect, however, was not statistically significant. It was impossible to calculate an overall PF for the other preventive measures, but the tendency of their caries-inhibiting effect was described. The use of toothpaste and gel with a high fluoride concentration of $1500-5000 \mathrm{ppm}$ or of complementary chlorhexidine during orthodontic treatment showed a demineralisation-inhibiting tendency. The use of a polymeric tooth coating on the tooth surface around the brackets showed almost no demineralisation-inhibiting effect. Conclusions Many publications had to be excluded from this systematic review because of inadequate research designs. Future clinical trials are needed to give evidence-based advice on the optimal caries-prevention strategy.

\begin{abstract}
Commentary
One of the risks of fixed orthodontic treatment is decalcification or frank caries occurring as a result of poor oral hygiene and plaque accumulation during treatment. The presence of fixed orthodontic attachments and auxiliaries makes plaque control more difficult and the process of demineralisation and cavitation can be rapid. Much orthodontic treatment takes place during the adolescent years when attention to oral hygiene may be less than fastidious, coupled with a significant intake of cariogenic food and drink. Orthodontic treatment with fixed appliances generally takes 18-22 months to complete. Although good oral hygiene is a prerequisite for fixed appliance therapy, plaque control can vary during this time. Most orthodontists advocate a nightly fluoride mouth rinse to supplement conventional cleaning with toothbrushes and fluoridated toothpaste. Some practitioners also advocate fluoride varnish or application of chlorhexidine or enamel sealing as well as fluoride-releasing elastomers and bracket bonding materials.

Until now, there has been no study that compared the effectiveness of these measures. This systematic review aims to develop evidence-based recommendations for prevention of demineralisation and caries associated with fixed appliances.
\end{abstract}

Address for correspondence: Dr A Derks, Department of Orthodontics and Oral Biology, University Medical Centre, Nijmegen, The Netherlands. E-mail: a.derks@dent.umcn.nl
A search for publications related to prevention of demineralisation and fixed appliance treatment was carried out using both Medline and Pubmed. Only RCT were included where the preventive measure was used throughout the duration of orthodontic treatment, and where demineralisation was scored in both treatment and control groups. Following this, 15 publications were included in the systematic review, divided into four groups demonstrating different preventive measures. These were group A for fluoride, group B for chlorhexidine, group $\mathrm{C}$ for sealants and group $\mathrm{D}$ for bonding materials.

The PF was used to quantify the caries-inhibiting effect of each of these measures. PF expresses a relationship between the incidence of demineralisation in the control group compared with the experimental group. The 95\% confidence intervals (CI) were also presented where possible, and details were provided of publications where data was insufficient. The authors explained that, although a meta-analysis of RCT would have been ideal, only a systematic review could be carried out for this study. This was because of the lack of publications fulfilling the inclusion criteria, lack of homogeneity and insufficient data allowing calculation of the 95\% CI.

This investigation concluded that, with the data available and with differences in trial designs, it was impossible to calculate PF values in groups A, B and C. An overall PF could be calculated in group $\mathrm{D}$. The latter included fluoride-releasing composites and glass ionomers from publications dating between 1992 and 2000. An overall PF of $20 \%$ for fluoride-releasing bonding agents was calculated, but the effect was not statistically significant. Although it was impossible to calculate overall PF in groups A, B and C, conclusions were drawn from the individual publications in these groups. These were that the use of toothpaste and gel with a high fluoride concentration $(1500-5000 \mathrm{ppm})$ or of complimentary chlorhexidine during orthodontic treatment with bonded fixed appliances showed a demineralisation-inhibiting tendency. The use of a polymeric coating on the tooth surface around the brackets or a fluoride-releasing material for bonding brackets showed almost no demineralisation-inhibiting effects.

As is generally the case with studies of this sort, the authors concluded that future RCT are still required to improve evidencebased advice to orthodontists on the optimal strategy for preventing decalcification during fixed orthodontic appliance treatment.

\section{Practice point}

- For orthodontic patients undergoing fixed appliance therapy, oral hygiene measures should include toothbrushing with fluoridated toothpaste, augmented by daily application of high fluoride concentration gel or chlorhexidine mouthwash. The efficacy of fluoride release from bonding materials or elastomers in reducing decalcification is unverified as yet.

\section{Julian O'Neill}

Kettering General Hospital NHS Trust, Kettering, Northamptonshire, UK

Evidence-Based Dentistry (2004) 5, 97.

doi: $10.1038 /$ sj.ebd. 6400300 